九州大学学術情報リポジトリ

Kyushu University Institutional Repository

\title{
An improved pattern matching algorithm for strings in terms of straight-line programs
}

Miyazaki, Masamichi

Department of Informatics, Kyushu University

Shinohara, Ayumi

Department of Informatics, Kyushu University

Takeda, Masayuki

Department of Informatics, Kyushu University

http://hdl. handle. net/2324/3236

出版情報 : DOI Technical Report. 130, 1997-01-23. Department of Informatics, Kyushu University バージョン：

権利関係 : 


\section{DOI Technical Report}

An improved pattern matching algorithm

for strings in terms of straight-line programs

Masamichi Miyazaki

Ayumi Shinohara

Masayuki Takeda

January 23, 1997

Department of Informatics

Kyushu University 33

Fukuoka 812-81, Japan 


\title{
An improved pattern matching algorithm for strings in terms of straight-line programs
}

\author{
Masamichi Miyazaki Ayumi Shinohara \\ Masayuki Takeda \\ \{masamich, ayumi, takeda\}@i.kyushu-u.ac.jp \\ Department of Informatics, Kyushu University 33, \\ Fukuoka 812-81, Japan
}

\begin{abstract}
We show an efficient pattern matching algorithm for strings that are succinctly described in terms of straight-line programs, in which the constants are symbols and the only operation is the concatenation. In this paper, both text $T$ and pattern $P$ are given by straight-line programs $\mathcal{T}$ and $\mathcal{P}$. The length of the text $T$ (pattern $P$, resp.) may grows exponentially with respect to its description size $|\mathcal{T}|=n(|\mathcal{P}|=m$, resp.). We show a new combinatorial property concerning with the periodic occurrences in a text. Based on this property, we develop an $O\left(n^{2} m^{2}\right)$ time algorithm using $O(n m)$ space, which outputs a compact representation of all occurrences of $P$ in $T$. This is superior to the algorithm proposed by Karpinski et al. [11], which runs in $O\left((n+m)^{4} \log (n+m)\right)$ time using $O\left((n+m)^{3}\right)$ space, and finds only one occurrence. Moreover, our algorithm is much simpler than theirs.
\end{abstract}

\section{Introduction}

The string pattern matching is a task to find all occurrences of a pattern in a text. In practice the text is large and is stored in secondary storage, hence most of the time required for pattern matching is devoted to data transmission. If the text is stored in some compressed form, the data transmission time is decreased according to the compression ratio. Text compression thus speeds up pattern-matching. Of course, the processing time (excluding I/O time) may be much longer than searching the original text. Therefore it is important to give an efficient pattern matching algorithm for searching a compressed text directly.

The problem of pattern matching in compressed text is of not only practical interest but also of theoretical interest. It has been studied recently by several researchers for several compression methods. For example, $[1,2,3,5,6]$ are for the run-length coding, [4] for the LZW coding, $[7,8,9]$ for the LZ77 coding.

A straight-line program is a compact representation of string. It is a context-free grammar in the Chomsky normal form that derives only one string. The length of the string represented by a straight-line program can be exponentially long with respect to the size of the straight-line program. In this sense, conversion of string into straight-line program can be viewed as a kind of text compressions. In fact, any text compressed by the LZW coding can be transformed directly into a straight-line program within a constant factor.

In this paper we concentrate on the pattern-matching problem where both text and pattern are represented in terms of straight-line programs. Karpinski et al.[10] showed the first 
polynomial-time algorithm. Later in [11] they proposed an $O\left((n+m)^{4} \log (n+m)\right)$ time algorithm using $O\left((n+m)^{3}\right)$ space, where $n$ and $m$ are the sizes of straight-line programs representing the text and the pattern, respectively. However, the algorithm is complicated and finds only one occurrence of pattern. In this paper we describe a new combinatorial property concerning with the periodic occurrences of pattern in text, and then present an $O\left(n^{2} m^{2}\right)$ time algorithm using $O(n m)$ space, which is based on this property. Our algorithm is simple and easy to understand, and outputs an $O(n)$ representation of all occurrences of pattern in text.

\section{Preliminary}

In this paper, both text and pattern are described in terms of straight-line programs. A straight-line program $\mathcal{R}$ is a sequence of assignments as follows:

$$
X_{1}=\operatorname{expr}_{1} ; X_{2}=\operatorname{expr}_{2} ; \ldots ; X_{n}=\operatorname{expr}_{n}
$$

where $X_{i}$ are variables and $\operatorname{expr}_{i}$ are expressions of the form:

- $\operatorname{expr}_{i}$ is a symbol of a given alphabet $\Sigma$, or

- $\operatorname{expr}_{i}=X_{\ell} \cdot X_{r}(\ell, r<i)$, where $\cdot$ denotes the concatenation of $X_{\ell}$ and $X_{r}$.

Denote by $R$ the string which is derived from the last variable $X_{n}$ of the program $\mathcal{R}$. The size of the straight-line program $\mathcal{R}$, denoted by $\|\mathcal{R}\|$, is defined to be the number of assignments in $\mathcal{R}$. The length of a string $w$ is denoted by $|w|$. We identify a variable $X_{i}$ with the string represented by $X_{i}$ if it is clear from the context.

Example 1 Let us consider the following straight-line program $\mathcal{R}$ :

$$
\begin{gathered}
X_{1}=\mathrm{a} ; X_{2}=\mathrm{b} ; X_{3}=X_{1} \cdot X_{2} ; X_{4}=X_{3} \cdot X_{1} ; X_{5}=X_{3} \cdot X_{4} ; \\
X_{6}=X_{5} \cdot X_{5} ; X_{7}=X_{4} \cdot X_{6} ; X_{8}=X_{7} \cdot X_{5} .
\end{gathered}
$$

We can see that $R=X_{8}=$ abaababaababaababa, and $\|\mathcal{R}\|=8,|R|=18$. The evaluation tree is shown in Figure 1.

We introduce a measure depth of a variable $X$ in a straight-line program $\mathcal{R}$ defined by

$$
\operatorname{depth}(X)= \begin{cases}1 & \text { if } X=a \in \Sigma \\ 1+\max \left(\operatorname{depth}\left(X_{\ell}\right), \operatorname{depth}\left(X_{r}\right)\right) & \text { if } X=X_{\ell} \cdot X_{r} .\end{cases}
$$

It corresponds to the length of the longest path from the node $X$ to a leaf in the tree.

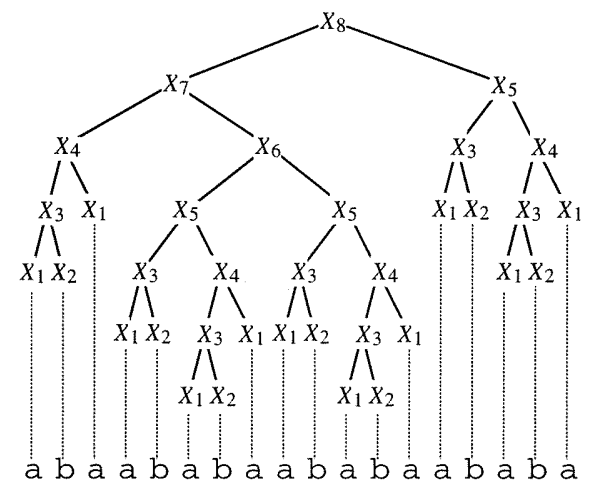

Figure 1: Evaluation tree of $\mathcal{R}$ in Example 1. 


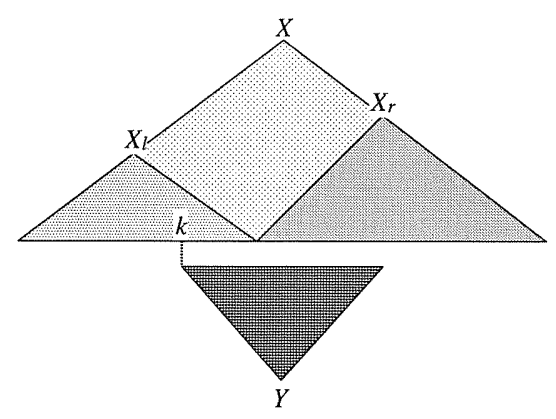

Figure 2: $k \in O c c^{\star}(X, Y)$, since $\mathrm{Y}$ covers the boundary between $X_{\ell}$ and $X_{r}$.

For a string $w$ denote by $w[f . . t](1 \leq f \leq t \leq|w|)$ the subword of $w$ starting at $f$ and ending at $t$. The pattern matching problem for strings in terms of straight-line programs is, given straight-line programs $\mathcal{P}$ and $\mathcal{T}$ which are the descriptions of pattern $P$ and text $T$ respectively, to find all occurrences of $P$ in $T$. Namely, we will compute the following set:

$$
\operatorname{Occ}(T, P)=\{i: T[i . . i+|P|-1]=P\} .
$$

Hereafter, we use $X_{i}$ for a variable in $\mathcal{T}$ and $Y_{j}$ for a variable in $\mathcal{P}$. We also denote by $n$ and $m$ the sizes of $\mathcal{T}$ and $\mathcal{P}$, respectively. For a set $U$ of integers and an integer $k$, we denote $U \oplus k=\{i+k: i \in U\}$ and $U \ominus k=\{i-k: i \in U\}$.

\section{Overview of algorithm}

In this section, we give an overview of our algorithm together with its basic idea. Let $X$ be a variable which appears in $\mathcal{T}$, and $Y$ be a variable in $\mathcal{P}$. First we consider a compact representation of the set $\operatorname{Occ}(X, Y)$.

Suppose $X=X_{\ell} \cdot X_{r}$. We define $O c c^{\star}(X, Y)$ to be the set of occurrences of $Y$ in $X$ such that $Y$ covers the boundary between $X_{\ell}$ and $X_{r}$ (see Figure 2):

$$
O c c^{\star}(X, Y)=\left\{s \in O c c(X, Y):\left|X_{\ell}\right|-|Y|+1 \leq s \leq\left|X_{\ell}\right|+1\right\} .
$$

For the sake of convenience, let $O c c^{\star}(X, Y)=\operatorname{Occ}(X, Y)$ for $X=a \in \Sigma$. Then we have the following lemma, which is informally stated in [8].

Lemma 1 For any $X$ in $\mathcal{T}$ and any $Y$ in $\mathcal{P}, \operatorname{Occ}^{\star}(X, Y)$ forms a single arithmetic progression.

We have the following observation (see Figure $3(\mathrm{a})$ ):

Observation 1 (decomposition of text variables)

For $X_{i}=X_{\ell(i)} \cdot X_{r(i)}$ in $\mathcal{T}$ and $Y$ in $\mathcal{P}$,

$$
O c c\left(X_{i}, Y\right)=O c c^{\star}\left(X_{i}, Y\right) \cup O c c\left(X_{\ell(i)}, Y\right) \cup\left(O c c\left(X_{r(i)}, Y\right) \oplus\left|X_{\ell(i)}\right|\right) .
$$

The above observation suggests that $\operatorname{Occ}\left(X_{n}, Y\right)$ can be represented by a combination of $\left\{\operatorname{Occ}^{\star}\left(X_{i}, Y\right)\right\}_{i=1}^{n}$. By Lemma 1, each $O c c^{\star}\left(X_{i}, Y\right)$ forms a single arithmetic progression, which can be stored in $O(1)$ space as a triple of the first element, the last element, and the step of the progression. Thus the desired output, a compact representation of the set $\operatorname{Occ}(T, P)=\operatorname{Occ}\left(X_{n}, Y_{m}\right)$ is given as a combination of $\left\{O c c^{\star}\left(X_{i}, Y_{m}\right)\right\}_{i=1}^{n}$, which occupies $O(n)$ space. Moreover, as we will show in Lemma 4 in Section 5, the membership to the set $\operatorname{Occ}\left(X_{i}, Y_{j}\right)$ can be answered in $O\left(\operatorname{depth}\left(X_{i}\right)\right)=O(n)$ time using this representation. Therefore the computation of the set $\operatorname{Occ}(T, P)$ is reduced to the computation of each set $O c c^{\star}\left(X_{i}, Y_{m}\right), i=1, \ldots, n$. The next observation gives us a recursive procedure to compute the set $O c c^{\star}\left(X_{i}, Y_{j}\right)$ (see Figure $3(\mathrm{~b})$ ): 


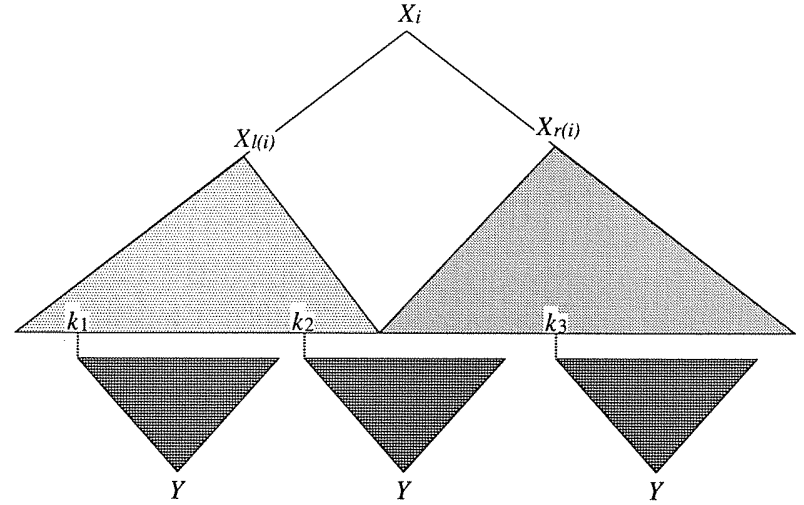

(a) $k_{1}, k_{2}, k_{3} \in \operatorname{Occ}\left(X_{i}, Y\right)$, while $k_{1} \in$ $O c c\left(X_{\ell(i)}, Y\right), \quad k_{2} \in O c c^{\star}\left(X_{i}, Y\right)$, and $k_{3}-$ $\left|X_{\ell(i)}\right| \in \operatorname{Occ}\left(X_{r(i)}, Y\right)$.
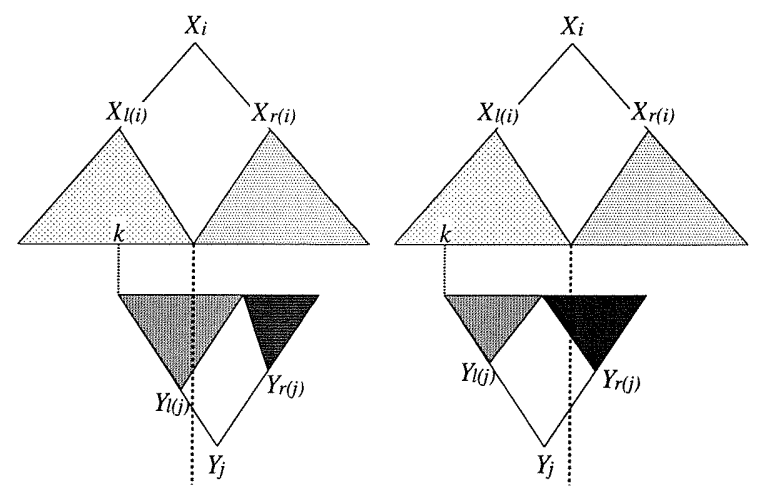

(b) $k \in \operatorname{Occ}^{\star}\left(X_{i}, Y_{j}\right)$ if and only if either $k \in$ $O c c^{\star}\left(X_{i}, Y_{\ell(j)}\right)$ and $k+\left|Y_{\ell(j)}\right| \in O c c\left(X_{i}, Y_{r(j)}\right)$ (left case), or $k \in O c c\left(X_{i}, Y_{\ell(j)}\right)$ and $k+\left|Y_{\ell(j)}\right| \in$ $O c c^{\star}\left(X_{i}, Y_{r(j)}\right)$ (right case).

Figure 3: Decomposition of variables.

\section{Observation 2 (decomposition of pattern variables)}

For $X_{i}$ in $\mathcal{T}$ and $Y_{j}=Y_{\ell(j)} \cdot Y_{r(j)}$ in $\mathcal{P}$,

$$
\begin{aligned}
& O c c^{\star}\left(X_{i}, Y_{j}\right)=O c c_{\ell}^{\star}\left(X_{i}, Y_{j}\right) \cup O c c_{r}^{\star}\left(X_{i}, Y_{j}\right), \text { where } \\
& O \operatorname{Occ}_{\ell}^{\star}\left(X_{i}, Y_{j}\right)=\operatorname{Occ}\left(X_{i}, Y_{\ell(j)}\right) \cap\left(\operatorname{Occ}\left(X_{i}, Y_{r(j)}\right) \ominus\left|Y_{\ell(j)}\right|\right), \text { and } \\
& O c c_{r}^{\star}\left(X_{i}, Y_{j}\right)=\operatorname{Occ}\left(X_{i}, Y_{\ell(j)}\right) \cap\left(\operatorname{Occ}^{\star}\left(X_{i}, Y_{r(j)}\right) \ominus\left|Y_{\ell(j)}\right|\right) \text {. }
\end{aligned}
$$

The problem to be overcome is to perform the set operations, union and intersection efficiently, since each set may possibly contain exponentially many elements.

Lemma 2 in the next section is a key to solving this problem. The key lemma concerns with the periodicities in strings. It guarantees that each of $O c c_{\ell}^{\star}\left(X_{i}, Y_{j}\right)$ and $O c c_{r}^{\star}\left(X_{i}, Y_{j}\right)$ forms a single arithmetic progression again. This enables us to perform the union operation of these two sets in $O(1)$ time. At the same time, the key lemma gives us a basis to construct an efficient procedure of computing $O c c_{\ell}^{\star}\left(X_{i}, Y_{j}\right)$ from $O c c^{\star}\left(X_{i}, Y_{\ell(j)}\right)$, assuming the function FirstMismatch which returns the first position of the mismatches between $X_{i}$ and $Y_{r(j)}$. We can compute the set $O c c_{r}^{\star}\left(X_{i}, Y_{j}\right)$ in the same way. In Section 5, we will explain these procedures in detail.

When computing each $O c c^{\star}\left(X_{i}, Y_{j}\right)$ recursively, we may often refer to the same set $O c c^{\star}\left(X_{i^{\prime}}, Y_{j^{\prime}}\right)$ repeatedly for $i^{\prime}<i$ and $j^{\prime}<j$. We take the dynamic programming strategy. Let us consider an $n \times m$ table $A p p$ where each entry $A p p[i, j]$ at row $i$ and column $j$ stores the triple representing the set $O c c^{\star}\left(X_{i}, Y_{j}\right)$. We compute each $A p p[i, j]$ in bottom-up manner, for $i=1, \ldots, n$ and $j=1, \ldots, m$. As we will show in Lemma 6 in Section 5 , each $A p p[i, j]$ is computable in $O\left(\operatorname{depth}\left(X_{i}\right) \cdot \operatorname{depth}\left(Y_{j}\right)\right)$ time. Since $\operatorname{depth}\left(X_{i}\right) \leq n$ and $\operatorname{depth}\left(Y_{j}\right) \leq m$ for any $X_{i}$ and $Y_{j}$, we can construct the whole table $A p p$ in $O\left(n^{2} m^{2}\right)$ time. The size of the whole table is $O(n m)$, since each triple occupies $O(1)$ space. Hence we have the main theorem of this paper.

Theorem 1 Given two straight-line programs $\mathcal{T}$ and $\mathcal{P}$, we can compute an $O(n)$ size representation of the set $\operatorname{Occ}(T, P)$ of all occurrences of the pattern $P$ in the text $T$, in $O\left(n^{2} m^{2}\right)$ time using $O(n m)$ work space. For this representation, the membership to the set Occ $(T, P)$ can be determined in $O(n)$ time. 


\section{Key lemma}

This section shows the key lemma on a property of periodic occurrences of a pattern in a text, which our algorithm based on. Let $T$ and $P$ be strings of a text and a pattern. At first we define the function FirstMismatch $(T, P, k)$ which returns the first (leftmost) position of mismatches, when we compare $P$ with $T$ at position $k$. Formally,

$$
\text { FirstMismatch }(T, P, k)=\min \{1 \leq i \leq|P|: T[k+i-1] \neq P[i]\},
$$

for $1 \leq k \leq|T|-|P|+1$. If there is no such $i$, the value of FirstMismatch $(T, P, k)$ is nil. The value is a witness of $k \notin O c c(T, P)$.

Lemma 2 (Key Lemma). Let $T=u^{p} z\left(u, z \in \Sigma^{+}, p \geq 0\right)$ and $P \in \Sigma^{+}$. The set $S=$ $\operatorname{Occ}(T, P) \cap\{1+i|u|: i=0,1, \ldots, p\}$ forms a single arithmetic progression, which can be computed by at most three calls of FirstMismatch.

Proof (sketch) We use the following notation in this proof: For two integers $a$ and $b$, we denote by $\langle q, r\rangle=\operatorname{div}(a, b)$ that $q$ is the quotient and $r$ is the remainder of the division of $a$ by $b$. That is, $a=b \cdot q+r$ and $0 \leq r<b$.

Let $h=\max \{j \leq|T|-|P|+1: j=1+i|u|$ for $i=0,1, \ldots, p\}$. If no such $j$ exist, $S=\phi$. The case $h \leq 1+2|u|$ is trivial, since $S$ contains at most three positions. We consider the case $h \geq 1+3|u|$. At the beginning, we invoke the function FirstMismatch for the two positions 1 and $h$ as follows:

$$
\begin{aligned}
& \text { miss } 1=\text { FirstMismatch }(T, P, 1), \text { and } \\
& \text { miss } 2=\text { FirstMismatch }(T, P, h) .
\end{aligned}
$$

Note that $1 \leq$ miss 1, miss $2 \leq|P|$, if not nil. It is convenient that we regard nil as $|P|+1$. Depending on the values of miss 1 and miss2, we have six cases as shown in Figure 4.

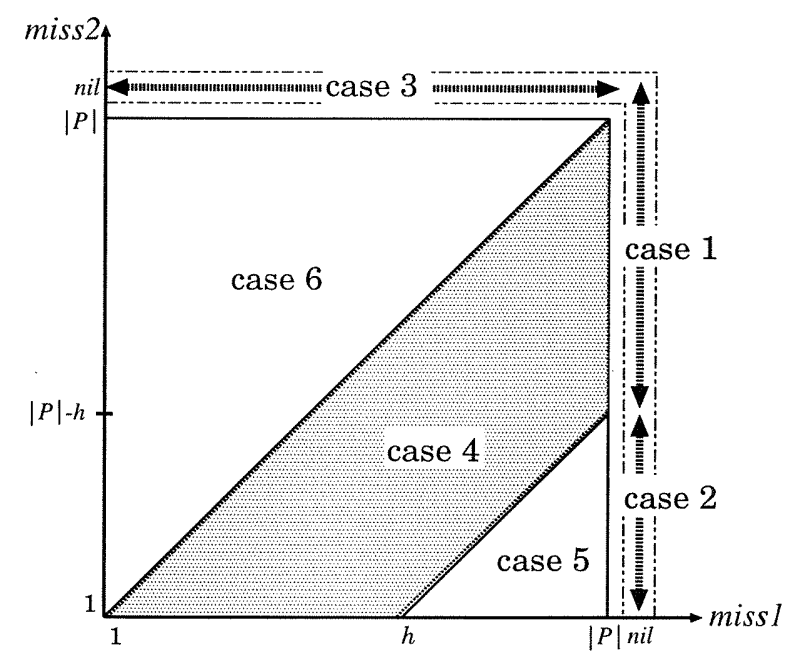

Figure 4: Six cases depending on miss1 and miss2.

(Cases 2 and 5 are vacant if $h>|P|$.)

case 1: miss $1=$ nil and miss $2=$ nil or $|P|-h+1<$ miss2. See Figure 5 .

Let $\langle q, r\rangle=\operatorname{div}(|P|,|u|)$ and $\left\langle q^{\prime}, r^{\prime}\right\rangle=\operatorname{div}(h+m i s s 2-2,|u|)$. We can show that $P=u^{q} u[1 . . r]$ and $T=u^{q^{\prime}} u\left[1 . . r^{\prime}\right] w$ for some $w \in \Sigma^{+}$with $u\left[r^{\prime}+1\right] \neq w[1]$. We can show that $S=\{1+i|u|: i \in\{0, . ., t\}\}$, where $t=q^{\prime}-q$ if $r^{\prime} \geq r$ and $t=q^{\prime}-q-1$ otherwise. We note that such $t$ can be directly computed by $\left\langle t, r^{\prime \prime}\right\rangle=\operatorname{div}(h+m i s s 2-|P|-2,|u|)$. 


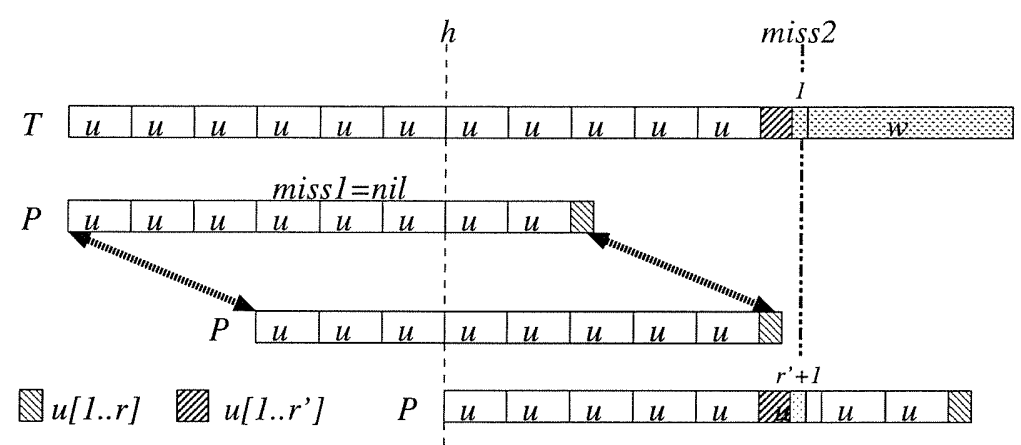

Figure 5: Case 1, $P=u^{q} u[1 . . r]$ and $T=u^{q^{\prime}} u\left[1 . . r^{\prime}\right] w$.

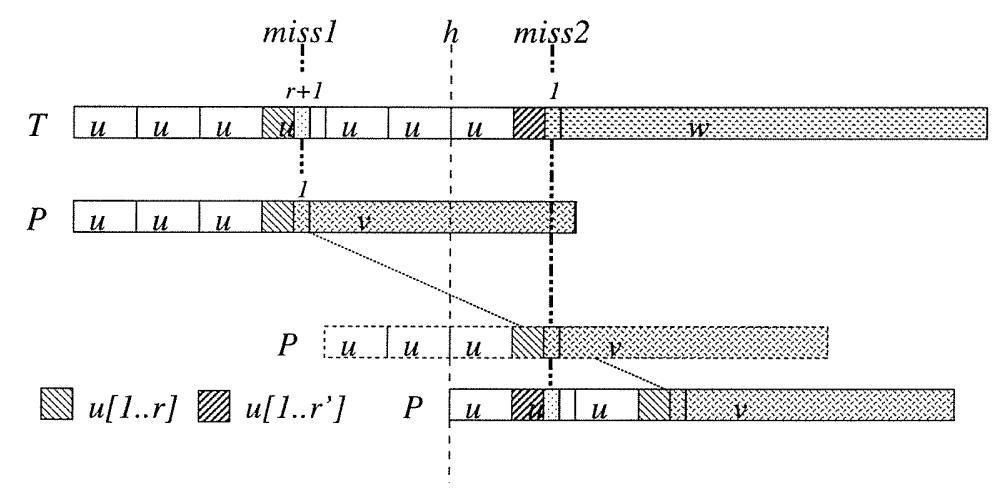

Figure 6: Case $4, P=u^{q} u[1 . . r] v$ and $T=u^{q^{\prime}} u\left[1 . . r^{\prime}\right] w$.

case 2: miss $1=$ nil and miss $2 \leq|P|-h+1$. (This is impossible if $h>|P|$ ).

Let $\langle q, r\rangle=\operatorname{div}(h+m i s s 2-2,|u|)$. We can show that $P=u^{q} u[1 . . r] v$ and $T=u^{q} u[1 . . r] w$ for some $v, w \in \Sigma^{+}$such that $u[r+1] \neq v[1]=w[1]$ and $v$ is a prefix of $w$. Thus we have $S=\{1\}$.

case 3: miss $1 \neq$ nil and miss $2=$ nil.

Let $\langle q, r\rangle=\operatorname{div}(\operatorname{miss} 1-1,|u|)$, and $\left\langle q^{\prime}, r^{\prime}\right\rangle=\operatorname{div}(h+m i s s 1-2,|u|)$. We can show that $r=r^{\prime}, P=u^{q} u[1 . . r] v$ and $T=u^{q^{\prime}} u[1 . . r] w$ for some $v, w \in \Sigma^{+}$such that $u[r+1] \neq v[1]$ and $v$ is a prefix of $w$. Thus we have $S=\{h\}$.

case 4: miss $1-h+1<$ miss $2<$ miss 1 . See Figure 6 .

Let $\langle q, r\rangle=\operatorname{div}(\operatorname{miss} 1-1,|u|)$ and $\left\langle q^{\prime}, r^{\prime}\right\rangle=\operatorname{div}(h+m i s s 2-2,|u|)$. We can show that $P=u^{q} u[1 . . r] v$ and $T=u^{q^{\prime}} u\left[1 . . r^{\prime}\right] w$ for some $v, w \in \Sigma^{+}$such that $u[r+1] \neq$ $v[1]$ and $u\left[r^{\prime}+1\right] \neq w[1]$. If $r=r^{\prime}$ and $v$ is a prefix of $w$, then $S$ is a singleton of $s=1+p|u|-m i s s 1+m i s s 2$. Otherwise $S=\phi$. That is, the only candidate for the elements in $S$ is $s$. We can verify whether $S=\{s\}$ or $S=\phi$ by the third call of FirstMismatch $(T, P, s)$.

case 5: miss $2 \leq$ miss $1-h+1$. (This is impossible if $h>|P|$ ).

Let $\langle q, r\rangle=\operatorname{div}(h+m i s s 2-2,|u|)$, and $s=$ miss $1-h-m i s s 2+2$. Since we can show that $P=u^{q} u[1 . . r] v$ and $T=u^{q} u[1 . . r] w$ for some $v, w \in \Sigma^{+}$such that $u[r+1] \neq v[1]=w[1]$ and $v[s] \neq w[s]$, we have $S=\phi$.

case 6: miss $1 \leq$ miss 2 .

Let $\langle q, r\rangle=\operatorname{div}(m i s s 1-1,|u|)$, and $\left\langle q^{\prime}, r^{\prime}\right\rangle=\operatorname{div}(h+m i s s 1-2,|u|)$. Let $s=$ miss $2-$ miss $1+1$. Since we can show that $r=r^{\prime}, P=u^{q} u[1 . . r] v$ and $T=u^{q^{\prime}} u[1 . . r] w$ for some $v, w \in \Sigma^{+}$such that $u[r+1] \neq v[1]$ and $v[s] \neq w[s]$, we have $S=\phi$. 
For any case, $S$ forms a single arithmetic progression, and we can compute its representation by calling FirstMismatch at most three times.

The above lemma is the heart of our algorithm. It gives us an efficient way of computing the periodic pattern occurrences in the set $O \operatorname{Oc}^{\star}\left(X_{i}, Y_{\ell(j)}\right)$, assuming that the function FirstMismatch can be realized efficiently. In the next section, we will give such a realization of FirstMismatch $(X, Y, k)$ for variables $X$ in $\mathcal{T}$ and $Y$ in $\mathcal{P}$.

\section{$5 \quad$ Algorithm in detail}

In this section, we explain the details on the algorithm. That is, how to compute each entry $A p p[i, j]$ of the table, which represents the set $\operatorname{Occ}^{\star}\left(X_{i}, Y_{j}\right)$. The computation is done in bottom-up manner.

If either $X_{i}$ or $Y_{j}$ is a symbol, we can compute the entry $A p p[i, j]$ in a trivial way. We show how to compute $A p p[i, j]$ for $X_{i}=X_{\ell(i)} \cdot X_{r(i)}$ and $Y_{j}=Y_{\ell(j)} \cdot Y_{r(j)}$, assuming that all preceding entries $A p p\left[i^{\prime}, j^{\prime}\right]$ for $i^{\prime}<i$ and $j^{\prime}<j$ are already computed. We can also assume that we know all lengths $\left|X_{i^{\prime}}\right|$ and $\left|Y_{j^{\prime}}\right|$. As we have explained in Section 3, the critical point is the computation of $O c c_{\ell}^{\star}\left(X_{i}, Y_{j}\right)=O c c^{\star}\left(X_{i}, Y_{\ell(j)}\right) \cap\left(O c c\left(X_{i}, Y_{r(j)}\right) \ominus\left|Y_{\ell(j)}\right|\right)$.

Lemma 3 Independently of the cardinality of the set $O c c^{\star}\left(X_{i}, Y_{\ell(j)}\right)$, we can compute the set $O c c_{\ell}^{\star}\left(X_{i}, Y_{j}\right)$ by using the function FirstMismatch $\left(X_{i}, Y_{r(j)}, k\right)$ at most three times.

Proof In case that the cardinality of the set $O c c^{\star}\left(X_{i}, Y_{\ell(j)}\right)$ is at most two, we can compute the set $O c c_{\ell}^{\star}\left(X_{i}, Y_{j}\right)$ easily: For each $s \in O c c^{\star}\left(X_{i}, Y_{\ell(j)}\right)$ we check whether or not $s \in \operatorname{Occ}\left(X_{i}, Y_{r(j)}\right) \ominus\left|Y_{\ell(j)}\right|$ by using FirstMismatch $\left(X_{i}, Y_{r(j)}, s+\left|Y_{\ell(j)}\right|\right)$.

For the case that $O c c^{\star}\left(X_{i}, Y_{\ell(j)}\right)$ contains more than two positions, we apply Lemma 2 as follows. Let $L$ and $R$ be the minimum and the maximum elements in $O c c^{\star}\left(X_{i}, Y_{\ell(j)}\right) \oplus\left|Y_{\ell(j)}\right|$, respectively (Figure 7). Let $d$ be the step of the arithmetic progression of $O c c^{\star}\left(X_{i}, Y_{\ell(j)}\right)$, and let $p=(R-L) / d$. Then we can see that the string $X_{i}\left[L . .\left|X_{i}\right|\right]$ is of the form $u^{p} z$, where $u$ is the suffix of $Y_{\ell(j)}$ of length $d$. By Lemma 2, we can compute the set

$$
S=\operatorname{Occ}\left(X_{i}\left[L . .\left|X_{i}\right|\right], Y_{r(j)}\right) \cap\{1,1+d, \ldots, 1+p \cdot d\}
$$

by calling the function FirstMismatch $\left(X_{i}, Y_{r(j)}, k\right)$ at most three times. Since

$$
\begin{aligned}
S \oplus(L-1) & =\left(\operatorname{Occ}\left(X_{i}\left[L . .\left|X_{i}\right|\right], Y_{r(j)}\right) \oplus(L-1)\right) & \cap & \{L, L+d, \ldots, L+p \cdot d\} \\
& =\operatorname{Occ}\left(X_{i}, Y_{r(j)}\right) & \cap & \left(O c c^{\star}\left(X_{i}, Y_{\ell(j)}\right) \oplus\left|Y_{\ell(j)}\right|\right),
\end{aligned}
$$

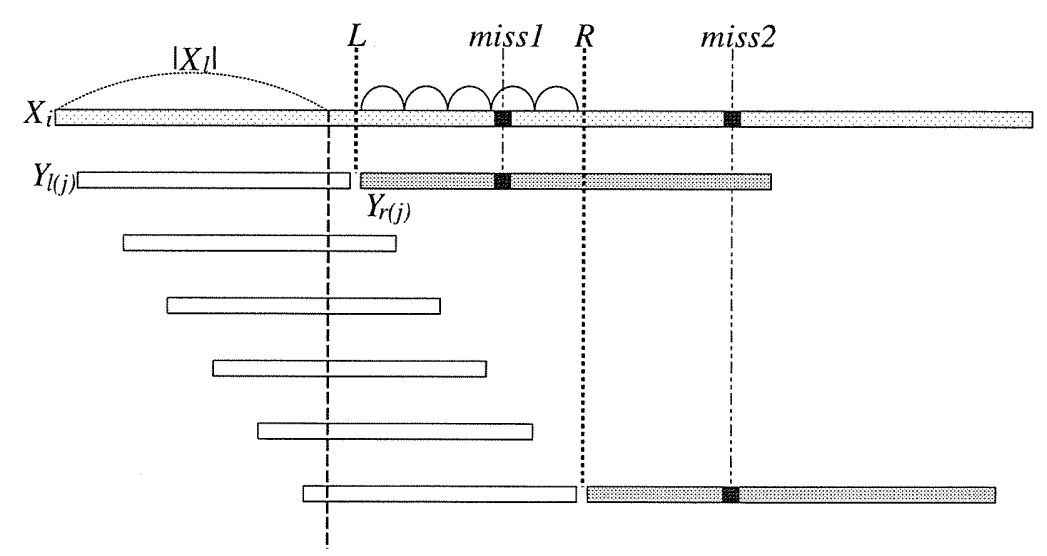

Figure 7: FirstMismatch $\left(X_{i}, Y_{r(j)}, L\right)$ and FirstMismatch $\left(X_{i}, Y_{r(j)}, R\right)$. 
function FirstMismatch $(X, Y, k)$ : integer;

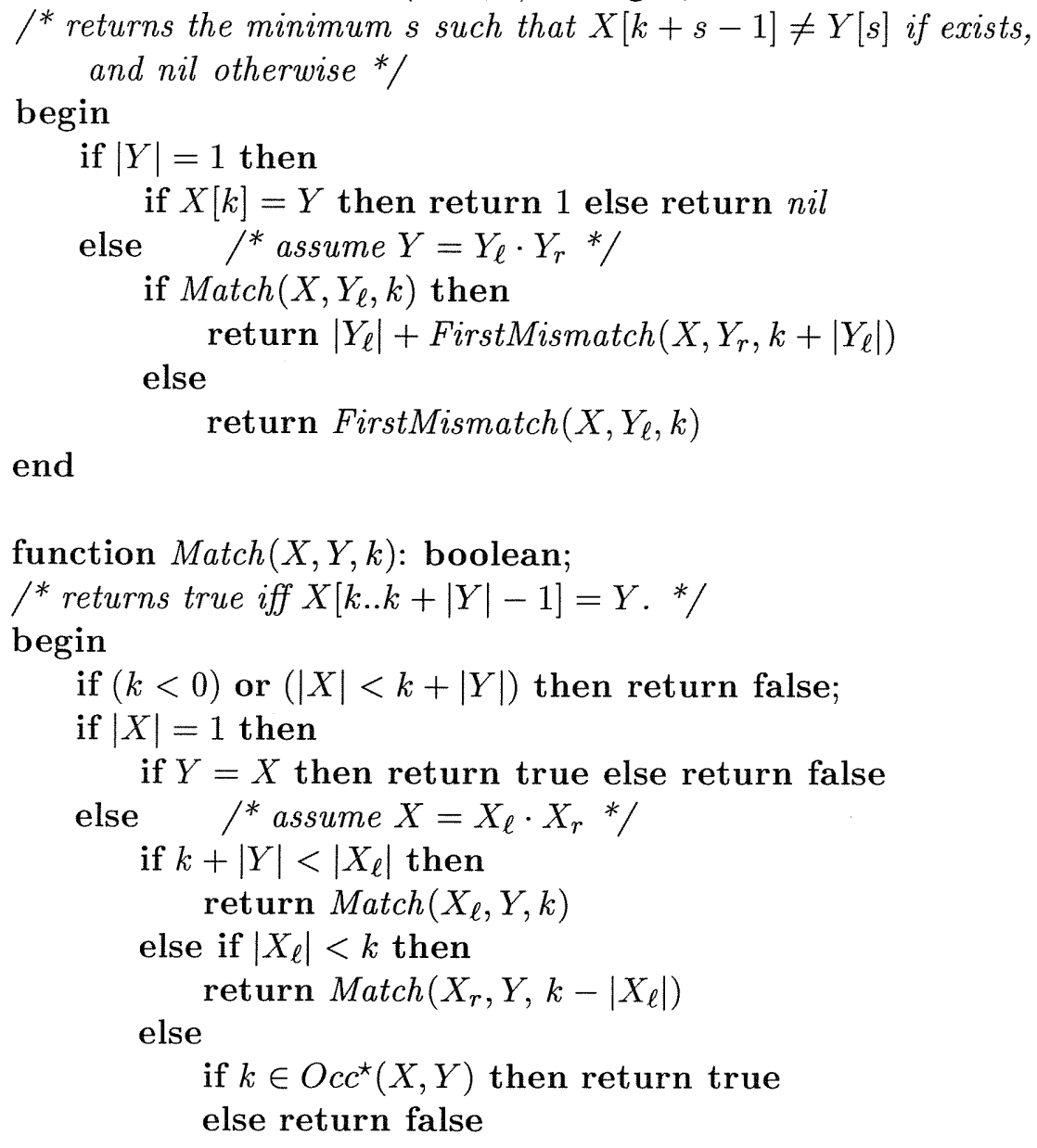

end

Figure 8: Pseudo-codes of the functions FirstMismatch and Match.

we have $S \oplus\left(L-1-\left|Y_{\ell(j)}\right|\right)=\left(O c c\left(X_{i}, Y_{r(j)}\right) \ominus\left|Y_{\ell(j)}\right|\right) \cap O c c^{\star}\left(X_{i}, Y_{\ell(j)}\right)=O \operatorname{Occ}_{\ell}^{\star}\left(X_{i}, Y_{j}\right)$, which is the desired set.

We show how to realize the function FirstMismatch $(X, Y, k)$ for each pair of variables $X$ in $\mathcal{T}$ and $Y$ in $\mathcal{P}$ and an integer $k$. Remark the following recursive property:

Observation 3 For two variables $X$ in $\mathcal{T}$ and $Y$ with $Y=Y_{\ell} \cdot Y_{r}$ in $\mathcal{P}$,

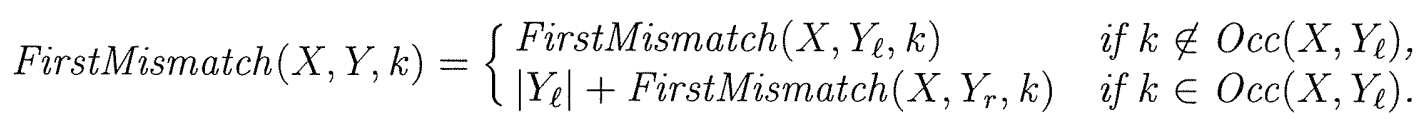

Figure 8 shows a pseudo-code of the function FirstMismatch, where the function $\operatorname{Match}(X, Y, k)$ returns true if and only if $k \in \operatorname{Occ}(X, Y)$. The correctness of $\operatorname{Match}(X, Y, k)$ is directly derived from Observation 1.

Lemma 4 The function $\operatorname{Match}\left(X_{i}, Y_{j}, k\right)$ answers in $O\left(\operatorname{depth}\left(X_{i}\right)\right)$ time.

Proof The membership query of the form $k \in O c c^{\star}\left(X_{i^{\prime}}, Y_{j^{\prime}}\right)$ can be answered in $O(1)$ time by simple calculations for any $i^{\prime}<i$ and $j^{\prime}<j$, since it is already computed and stored in 
the entry $A p p\left[i^{\prime}, j^{\prime}\right]$. Moreover, the number of recursive calls of $\operatorname{Match}\left(X_{i}, Y_{j}, k\right)$ is at most $\operatorname{depth}\left(X_{i}\right)$. Thus the lemma holds.

Lemma 5 The function FirstMismatch $\left(X_{i}, Y_{j}, k\right)$ answers in $O\left(\operatorname{depth}\left(X_{i}\right) \cdot \operatorname{depth}\left(Y_{j}\right)\right) \operatorname{time.}$

Proof We can verify easily that the number of recursive calls of FirstMismatch $\left(X_{i}, Y_{j}, k\right)$ is at most $\operatorname{depth}\left(Y_{j}\right)$. At each call, FirstMismatch $\left(X_{i}, Y_{j}, k\right)$ calls the function $\operatorname{Match}\left(X_{i}, Y_{j}, k\right)$ once. By Lemma 4 , it answers in $O\left(\operatorname{depth}\left(Y_{j}\right)\right)$ time. Thus the lemma holds.

By Lemma 3 and Lemma 5, we have the following result.

Lemma 6 We can compute each entry $A p p[i, j]$ in $O\left(\operatorname{depth}\left(X_{i}\right) \cdot \operatorname{depth}\left(Y_{j}\right)\right)$ time.

\section{Conclusion}

We have shown an improved pattern matching algorithm for strings in terms of straight-line programs. In Table 1, we summarize the results compared to the previous ones [10, 11], from the view points of time complexity, space complexity, and pattern detection ability when the pattern occurs in the text more than twice.

We briefly state the improvement of our algorithm compared to the that in [11]. The latter algorithm consists of two phases: At the first phase, it computes two sets: $\operatorname{Pref}\left(X_{i}, Y_{j}\right)$ of the lengths of prefixes of $Y_{j}$ that are suffixes of $X_{i}$, and $\operatorname{Suff}\left(X_{i}, Y_{j}\right)$ of the lengths of suffixes of $Y_{j}$ that are prefixes of $X_{i}$. At the second phases, it computes the set $\operatorname{Occ}\left(X_{i}, Y_{j}\right)$ from $\operatorname{Pref}\left(X_{i}, Y_{j}\right)$ and $\operatorname{Suff}\left(X_{i}, Y_{j}\right)$ by solving certain linear Diophantine equations with using Euclid's algorithm. Each $\operatorname{Suff}\left(X_{i}, Y_{j}\right)$ and $\operatorname{Pref}\left(X_{i}, Y_{j}\right)$ can be stored in $O\left(\operatorname{depth}\left(X_{i}\right)+\operatorname{depth}\left(Y_{j}\right)\right)$ space, although $\operatorname{Occ}\left(X_{i}, Y_{j}\right)$ occupies only $O(1)$ space. On the other hand, our algorithm directly computes $\operatorname{Occ}\left(X_{i}, Y_{j}\right)$. The property of periodic occurrences of a pattern in a text shown in the key lemma enabled the direct computation.

Both of these two algorithms use the information on the first position of mismatches. In our algorithm, pattern-to-text comparisons are enough, while the previous algorithm also requires text-to-text comparisons and pattern-to-pattern comparisons. This is the reason why the previous algorithm requires $(n+m) \times(n+m)$ table with $O(n+m)$ size entries in order to store the information on overlaps, while our algorithms requires only $n \times m$ table with $O(1)$ size entries. This is also the contribution of the key lemma.

Recently, Gassieniec et al. [8] have developed a series of efficient algorithms, including pattern matching algorithm, for strings in terms of composition systems. A composition system is an extension of the straight-line program, where substring selection is also allowed as well as concatenation, in order to simulate the LZ77 coding scheme. We will adapt our algorithm to treat composition systems in future works, hopefully combined with the randomized approaches [9].

Table 1: Summary

\begin{tabular}{|c|c|c|c|}
\hline algorithm & time & space & detection \\
\hline$[10]$ & $O\left((n+m)^{7}\right)$ & not estimated & some one \\
{$[11]$} & $O\left((n+m)^{4} \log (n+m)\right)$ & $O\left((n+m)^{3}\right)$ & some one \\
Ours & $O\left(n^{2} m^{2}\right)$ & $O(n m)$ & all \\
\hline
\end{tabular}




\section{References}

[1] A. Amir and G. Benson. Efficient two-dimensional compressed matching. In Proc. Data Compression Conference, page 279, 1992.

[2] A. Amir and G. Benson. Two-dimensional periodicity and its application. In Proc. 3rd Symposium on Discrete Algorithms, page 440, 1992.

[3] A. Amir, G. Benson, and M. Farach. Optimal two-dimensional compressed matching. In Proc. 21st International Colloquium on Automata, Languages and Programming, 1994.

[4] A. Amir, G. Benson, and M. Farach. Let sleeping files lie: Pattern matching in Zcompressed files. Journal of Computer and System Sciences, 52:299-307, 1996.

[5] A. Amir, G. M. Landau, and U. Vishkin. Efficient pattern matching with scaling. Journal of Algorithms, 13(1):2-32, 1992.

[6] T. Eilam-Tsoreff and U. Vishkin. Matching patterns in a string subject to multilinear transformations. In Proc. International Workshop on Sequences, Combinatorics, Compression, Security and Transmission, 1988.

[7] M. Farach and M. Thorup. String-matching in Lempel-Ziv compressed strings. In 27th ACM STOC, pages 703-713, 1995.

[8] L. Gąsieniec, M. Karpinski, W. Plandowski, and W. Rytter. Efficient algorithms for Lempel-Ziv encoding. In Proc. 4th Scandinavian Workshop on Algorithm Theory, volume 1097 of Lecture Notes in Computer Science, pages 392-403. Springer-Verlag, 1996.

[9] L. Ga̧sieniec, M. Karpinski, W. Plandowski, and W. Rytter. Randomized efficient algorithms for compressed strings: the finger-print approach. In Proc. Combinatorial Pattern Matching, volume 1075 of Lecture Notes in Computer Science, pages 39-49. SpringerVerlag, 1996.

[10] M. Karpinski, W. Rytter, and A. Shinohara. Pattern-matching for strings with short descriptions. In Proc. Combinatorial Pattern Matching, volume 637 of Lecture Notes in Computer Science, pages 205-214. Springer-Verlag, 1995.

[11] M. Karpinski, W. Rytter, and A. Shinohara. An efficient pattern-matching algorithm for strings with short descriptions. Nordic Journal of Computing, 1997. (to appear). 